REIHE COMPUTATIONAL INTELLIGENCE SONDERFORSCHUNGSBEREICH 531

Design und Management komplexer technischer Prozesse und Systeme mit Methoden der Computational Intelligence

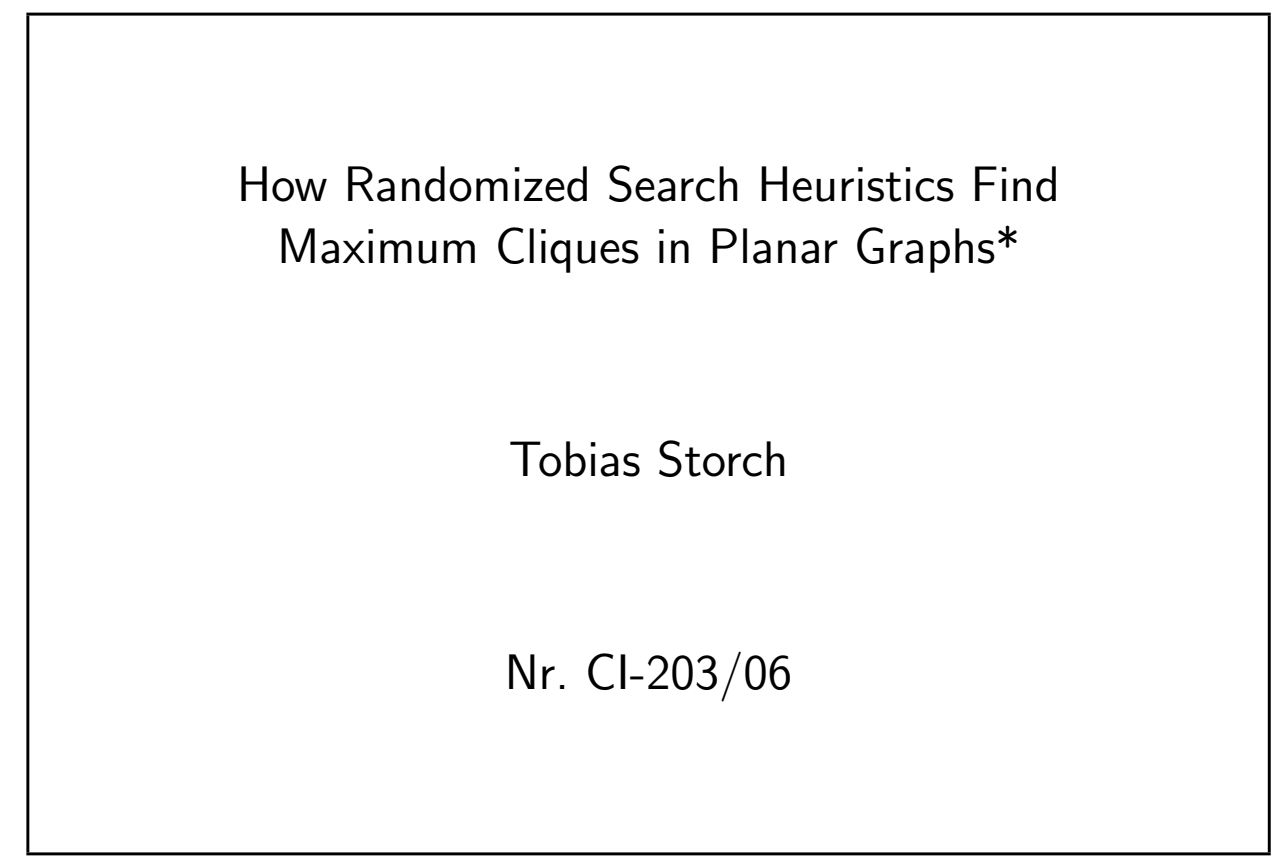

Interner Bericht

ISSN 1433-3325

March 2006

Sekretariat des SFB 531 . Universität Dortmund . Fachbereich Informatik/XI 44221 Dortmund · Germany

Diese Arbeit ist im Sonderforschungsbereich 531, „Computational Intelligence“, der Universität Dortmund entstanden und wurde auf seine Veranlassung unter Verwendung der ihm von der Deutschen Forschungsgemeinschaft zur Verfügung gestellten Mittel gedruckt. 


\title{
How Randomized Search Heuristics Find Maximum Cliques in Planar Graphs
}

\author{
Tobias Storch \\ Department of Computer Science 2 \\ University of Dortmund \\ 44221 Dortmund, Germany \\ tobias.storch@uni-dortmund.de
}

\begin{abstract}
Surprisingly, general search heuristics often solve combinatorial problems quite sufficiently, although they do not outperform specialized algorithms. Here, the behavior of simple randomized optimizers on the maximum clique problem on planar graphs is investigated rigorously. The focus is on the worst-, average-, and semi-average-case behaviors. In semi-random planar graph models an adversary is allowed to modify moderately a random planar graph, where a graph is chosen uniformly at random among all planar graphs. With regard to the heuristics particular interest is given to the influences of the following four popular strategies to overcome local optima: local- vs. global-search, single- vs. multi-start, small vs. large population, and elitism vs. non-elitism selection. Finally, the black-box complexities of the planar graph models are analyzed.
\end{abstract}

\section{Categories and Subject Descriptors}

F.2 [Theory of Computation]: Analysis of Algorithms and Problem Complexity

\section{General Terms}

Theory, Performance, Algorithms

\section{INTRODUCTION}

One of the best-known combinatorial optimization problems is to find a maximum clique in an undirected graph. A clique is a subset of vertices, where each two vertices are connected by an edge, and a maximal clique is a clique that is not contained in any larger clique. Finally, a maximum clique is a clique of largest size. The task to find a maximum clique is of practical and theoretical importance. This problem was among the first problems proven to be NP-hard [9]. However, on a wide range of real-world inputs a remarkable experimental success of randomized search heuristics and hybrid algorithms were reported [1]. The probably

\footnotetext{
* This work was supported in part by the Deutsche Forschungsgemeinschaft (DFG) as part of the Collaborative Research Center "Computational Intelligence" (SFB 531).
}

best-known types of the broad class of such optimizers are randomized local searches (RLSs), evolutionary algorithms (EAs), and Metropolis algorithms (MAs). Their variety is as huge as their area of applications. Here, we concentrate on the rigorous analysis of simple and general heuristics coming up without problem-specific components.

There exist several common ways to cope with the worstcase complexity of the maximum clique problem. One possibility is to investigate subclasses of all graphs. The probably best-known class is that of all (simple) planar graphs such graphs can be drawn in a plane without edges crossing. Several linear time algorithms were developed which find a maximum clique in a planar graph [5]. Another possibility to cope with the worst-case complexity of the maximum clique problem is to investigate random graphs models - such models are defined by probability distributions on all graphs. All these models are supposed to represent real-world inputs in a more appropriate manner than often artificial worst-case instances. The two approaches can be combined easily. Thus, beside planar graphs we investigate random planar graphs. Let $\mathcal{G}_{n}$ denote a graph drawn uniformly at random among all planar graphs on $n$ vertices $v_{1}, \ldots, v_{n}$. Simple expected (w.r.t. the random input) constant time algorithms find a maximum clique in $\mathcal{G}_{n}$ [10]. However, real-world instances are generally not as well-formed as random ones. In order to enrich and to robustify random input models, semi-random input models are considered, where two main variants exists. The first variant allows an adversary to present an arbitrary input, but this is modified moderately at random. The second variant presents a random input, but an adversary is allowed to vary it within limits [6]. These models generate combinations of worst- and average-case instances and moreover, semi-random input models are typically more adequate to distinguish between naïve and more sophisticated algorithms. We investigate three types of semi-random planar graphs. If an adversary modifies $\mathcal{G}_{n}$ by inserting or removing arbitrary edges, but keeps the graph planar, the resulting graph is denoted by $\mathcal{G}_{n}^{+}$and $\mathcal{G}_{n}^{-}$respectively. If the existence of at most $\varepsilon n$ edges of $\mathcal{G}_{n}$ is changed, for an arbitrarily small constant $\varepsilon>0$, but the graph is kept planar again, the resulting graph is denoted by $\mathcal{G}_{n}^{\star}$. $\mathcal{G}_{n}$ consists of random edges, while $\mathcal{G}_{n}^{+}, \mathcal{G}_{n}^{-}$, and $\mathcal{G}_{n}^{\star}$ contain some.

To understand the heuristics' and operators' successes especially contrasting the worst-, average-, and semi-average-case behaviors -, working principles, and the considered problems' structure are the major motivations. Theoretically only little is known about the behavior of simple randomized search heuristics on the maximum clique problem. 
Since general heuristics are intended to optimize objective functions $f: S \rightarrow \mathbb{Z}$ such a fitness function has to be designed for the clique problem. The search space $S=\{0,1\}^{n}$ is a canonical choice, where an element is interpreted as characteristic vector of the graph's vertices $V$ in an arbitrary order. Using the size of the clique as function value, if the subset represents a clique, the aim is maximization. Using $-\infty$ as function value, if the subset does not represent a clique, the optimizers need to be directed to a clique; typically by an initialization with (the element representing) the empty clique, namely the bit-string of $n$ zeros $0^{n}$ [8]. Thus, we consider the following objective function for a graph $G$, where $V=\left\{v_{1}, \ldots, v_{n}\right\}$ :

$$
f_{G}(x):= \begin{cases}|x| & \text { if }\left\{v_{i} \mid x_{i}=1,1 \leq i \leq n\right\} \text { is } \quad \text { a clique } \\ -\infty & \text { if }\left\{v_{i} \mid x_{i}=1,1 \leq i \leq n\right\} \text { is not a clique }\end{cases}
$$

Here, it is $|x|:=\sum_{i=1}^{n} x_{i}$ for a bit-string $x=x_{1} \cdots x_{n}$. The objective function $f_{G}$ can be evaluated efficiently.

A randomized algorithm is (typically) called efficient on a sequence $f=\left(f_{1}, \ldots, f_{n}, \ldots\right)$ of pseudo-Boolean functions $f_{n}:\{0,1\}^{n} \rightarrow \mathbb{Z}, n \in \mathbb{N}$, if the algorithm's expected number of function evaluations is upper bounded by a polynomial (in $n$ ) until first an optimal element is queried. Only counting the function evaluations is motivated by the observation that these require most resources during the optimization.

At first, let us take a closer look to the probably most simple randomized search heuristic, namely the (1+1) RLS (Randomized Local Search), and arbitrary planar graphs.

\section{Algorithm 1. (1+1) RLS}

1. Let $x:=0^{n}$.

2. Create $y$ by flipping one bit in $x$ chosen uniformly at random.

3. If $f(y) \geq f(x)$ let $x:=y$.

4. Goto 2 .

We begin with an investigation of the planar graph $G=$ $\left(V,\left\{\left\{v_{1}, v_{2}\right\}\right\}\right)$, where the only maximum clique is $\left\{v_{1}, v_{2}\right\}$. In the first step, the $(1+1)$ RLS creates each 1-clique with probability $1 / n$. Thus, one of the 1 -cliques $\left\{v_{3}\right\}, \ldots,\left\{v_{n}\right\}$ is created with probability $(n-2) / n=1-o(1)$. In this situation, the $(1+1)$ RLS is getting trapped in a local optimum since no change of a single bit leads to an element which has at least the same function value. This results in an expected - and even with high probability - number of infinite steps of the $(1+1)$ RLS on $G$. Since this specific planar graph $G$ is chosen with a positive probability by a uniform selection among all planar graphs the $(1+1)$ RLS needs an expected (w.r.t. the random bits of the algorithm and the random input) infinite number of steps on random planar graphs as well. We observe the $(1+1)$ RLS probable stagnates in local optima, here. There exist several common ways to get out of local optima. The probably best-known strategies are

- the application of a mutation operator in the algorithm (global search),

- the occasional algorithm's restart (multi-start strategy),

- the application of a population in the algorithm (large population), and

- the occasional algorithm's acceptance of a worse element (non-elitism selection).
Simple instances of optimizers applying these four strategies and modifying the $(1+1)$ RLS just slightly are defined and analyzed on planar and random planar graphs in Sections 3, 4, 5, and 6 respectively. Here, we determine which choices of the algorithms' parameter lead to an efficient locating of a maximum clique in planar graphs and which do not. The focus is on the identification of the optimal parameters settings and the resulting asymptotically exact number of steps needed for optimization. In Section 7 we define and consider the black-box complexities for finding a maximum clique in planar and random planar graphs. In Section 8 we describe how the investigations concerning planar and random planar graphs can be combined for the results on the semi-random planar graph models. Surprisingly, it will be pointed out that the Metropolis algorithm studied in Section 6 is an asymptotically optimal general randomized search heuristic for all types of planar graphs. We finish with some conclusions in Section 9, but we begin with some preliminaries in Section 2 .

\section{PRELIMINARIES}

We begin with upper bounds on the number (\#) of $k$ cliques in planar graphs.

Lemma 2. Let $G$ be a planar graph on $n, n \geq 3$, vertices.

\begin{tabular}{|r||l|l|l|l|l|}
\hline$k=$ & 0 & 1 & 2 & 3 and 4 & 5 and more \\
\hline$\# k$-cliques $\leq$ & 1 & $n$ & $3 n-6$ & $6 n-12$ & 0 \\
\hline
\end{tabular}

Proof. The graph $G$ consists of the 0 -clique and all $n 1$ cliques. By Euler's formula the number of 2-cliques is upper bounded by $3 n-6$ for $n \geq 3$ and each planar graph contains a vertex $v$ with degree $d \leq 5$. We successively remove such a vertex including its incident edges and observe that at most $2 d 3$-cliques and 4-cliques are removed, too. The number of 3- and 4-cliques removed is upper bounded by $\left(\begin{array}{l}d \\ 2\end{array}\right) \leq 2 d$ and $\left(\begin{array}{l}d \\ 3\end{array}\right) \leq 2 d$ respectively, where the inequalities hold for $d \leq 5$. Since the number of edges is upper bounded by $3 n-6$, the number of 3- and 4-cliques is upper bounded by $2 \cdot(3 n-6)=6 n-12$. By Kuratowski's theorem there are no cliques of size five or more in planar graphs.

We remark by Lemma 2 each planar graph consists of at most $16 n-29$ cliques.

We call $V^{\prime} \subseteq V$ loosely connected in $G$, if the number of edges $\{u, v\}$, where $u \in V^{\prime}$ and $v \in V \backslash V^{\prime}$, is at most one.

Lemma 3. Let $c>0$ be a small enough constant.

$\operatorname{Pr}\left[\mathcal{G}_{n}\right.$ contains $<c n$ loosely connected 3 -cliques $]<e^{-c n}$

$\operatorname{Pr}\left[\mathcal{G}_{n}\right.$ contains $<c n$ loosely connected 4 -cliques $]<e^{-c n}$

Proof. Since the complete graphs on three and four vertices are planar, the result follows by [10, Theorem 4.1].

Throughout this paper let $V_{3}$ and $V_{4}$ consist of all vertices of loosely connected 3- and 4-cliques respectively. Moreover, let $V_{3}^{\prime} \subseteq V_{3}$ and $V_{4}^{\prime} \subseteq V_{4}$ consist of all vertices of $V_{3}$ or $V_{4}$ respectively which are not connected to any vertex of $V \backslash V_{3}$ and $V \backslash V_{4}$ respectively. By definition of loosely connected 3- and 4-cliques it holds $\left|V_{3}^{\prime}\right| \geq\left|V_{3}\right|-\left|V_{3}\right| / 3=2\left|V_{3}\right| / 3 \geq 2 \mathrm{cn}$ and $\left|V_{4}^{\prime}\right| \geq\left|V_{4}\right|-\left|V_{4}\right| / 4=3\left|V_{4}\right| / 4 \geq 3$ cn respectively.

\section{GLOBAL SEARCH}

Let us define the simple $(1+1) \mathrm{EA}_{p}$ applying a mutation operator flipping each bit with probability $p$ in each step [3]. Obviously, it should hold $0<p<1$. 
Algorithm 4. $(\mathbf{1}+\mathbf{1}) \mathbf{E A}_{\boldsymbol{p}}$

1. Let $x:=0^{n}$

2. Create $y$ by flipping each bit in $x$ independently with probability $p$.

3. If $f(y) \geq f(x)$ let $x:=y$.

4. Goto 2 .

We observe each element is created in each step with a positive probability and the $(1+1) \mathrm{EA}_{1 / n}$ flips an expected number of one bit in each step - equivalently to the $(1+1)$ RLS.

Let us investigate planar graphs.

Lemma 5. Let $0<p \leq 1 / 2$ and $n$ be large enough.

1. Let $G$ be a planar graph. The $(1+1) \mathrm{EA}_{p}$ creates a maximum clique of $G$ within an expected number of $\mathcal{O}\left(e^{n p}(1+n p) / p^{6}\right)$ steps.

2. There exist planar graphs $G$, where the $(1+1) \mathrm{EA}_{p}$ needs an expected number of $\Omega\left(e^{n p} / p^{6}\right)$ steps to create a maximum clique of $G$.

The optimal parameters for the $(1+1) \mathrm{EA}_{p}$ are $p=\Theta(1 / n)$, where the expected number of steps is bounded by $\Theta\left(n^{6}\right)$, and the $(1+1) \mathrm{EA}_{p}$ is efficient, iff $p=n^{-\mathcal{O}(1)}$ and $p=$ $\mathcal{O}(\log n) / n$.

Proof. Part 1. Let $C$ be a maximum clique of $G$. If the element of the population $x$ does not represent a maximum clique, by Lemma 2 at least one specific mutation flipping at most $|x|+(|C|-1) \leq 6$ bits generates an individual which represents a subset of size at least $|C|-1$ of $C$. Its probability is lower bounded by $p^{6}(1-p)^{n-6}=\Omega\left(e^{-n p} \cdot p^{6}\right)$. In this situation, (a) the probability to create a maximum clique is lower bounded by $p^{+}:=p^{1}(1-p)^{n-1}=\Omega\left(e^{-n p} \cdot p\right)$ since one specific 1-bit mutation generates $C$. And moreover, (b) the probability to create a $(|C|-1)$-clique which is not a subset of $C$ is upper bounded by $p^{-}:=(6 n-12) \cdot p^{2}(1-p)^{n-2}=$ $\mathcal{O}\left(e^{-n p} \cdot p^{2} n\right)$ by Lemma 2 and since at least two bits have to change. Hence, the probability to perform (a) prior to (b) is bounded by $p^{+} /\left(p^{+}+p^{-}\right)$. In the case of a failure, we can repeat the argumentation. An expected number of $\left(p^{+}+p^{-}\right) / p^{+}=1+\mathcal{O}(n p)$ repetitions has to be performed. This leads to an expected number of $\mathcal{O}\left(e^{n p} / p^{6}\right) \cdot \mathcal{O}(1+n p)=$ $\mathcal{O}\left(e^{n p}(1+n p) / p^{6}\right)$ steps, in total.

Part 2. We define the investigated planar graph $G$. Therefore, let $V_{0}:=\left\{v_{1}, \ldots, v_{4}\right\}, V_{1}:=\left\{v_{5}, \ldots, v_{7}\right\}$, and $E:=$ $\left\{\{v, w\} \mid v, w \in V_{0}\right.$ or $\left.v, w \in V_{1}\right\}$. If the element represents the 0 -clique or a 1-clique on $V \backslash\left(V_{0} \cup V_{1}\right)$, then (a) with a probability of at least $p^{1}(1-p)^{n-1}$ and $p^{2}(1-p)^{n-2}$ respectively, a non-empty subset of the maximal clique $V_{1}$ is created. And moreover, (b) with a probability of at most $\left(2^{4}-1\right) \cdot p^{1}(1-p)^{n-1}$ and $\left(2^{4}-1\right) \cdot p^{2}(1-p)^{n-2}$ respectively, one of the non-empty cliques on the maximum clique $V_{0}$ is generated. However, the probability to perform (a) prior to (b) is bounded by $\Omega(1)$. In this situation, the individual represents a clique which is a subset of $V_{1}$ of size at least one. Afterwards, the probability to create a larger clique which is a subset of $V_{1}$ prior to perform (b) is bounded by $\Omega(1)$. Thus, with a probability of at least $\Omega(1) \cdot \Omega(1)=\Omega(1)$ the 3 -clique $V_{1}$ is created prior to a nonempty subset of $V_{0}$. Afterwards, the probability is bounded by $\left(\begin{array}{l}4 \\ 3\end{array}\right) p^{6}(1-p)^{n-6}+\left(\begin{array}{l}4 \\ 4\end{array}\right) p^{7}(1-p)^{n-7}=\mathcal{O}\left(e^{-n p} \cdot p^{6}\right)$ to create a 3 -clique which is contained in a 4 -clique or a 4-clique itself. This leads to an expected number of $\Omega(1) \cdot \Omega\left(e^{n p} / p^{6}\right)=$ $\Omega\left(e^{n p} / p^{6}\right)$ steps, in total.
Let us investigate random planar graphs.

Lemma 6. Let $0<p \leq 1 / 2$ and $n$ be large enough. The following holds with probability $1-e^{-\Omega(n)}$ and in expectation (w.r.t. the random input).

1. The $(1+1) \mathrm{EA}_{p}$ creates a maximum clique of $\mathcal{G}_{n}$ within an expected (w.r.t. the random bits of the algorithm) number of $\mathcal{O}\left(e^{n p}(1+n p) /\left(n p^{6}\right)\right)$ steps.

2. The $(1+1) \mathrm{EA}_{p}$ needs an expected (w.r.t. the random bits of the algorithm $)$ number of $\Omega\left(e^{n p} /\left(n p^{6}(1+n p)^{2}\right)\right)$ steps to create a maximum clique of $\mathcal{G}_{n}$.

The optimal and the parameters, where the $(1+1) \mathrm{EA}_{p}$ is efficient, are the same than on planar graphs.

Proof. By Lemma 3 with a failure probability of at most $e^{-c n}+e^{-c n}=e^{-\Omega(n)}$, for a constant $c>0$, the random planar graph $\mathcal{G}_{n}$ contains at least $c n$ loosely connected 3and 4-cliques. Let us assume that this holds in the following.

Part 1. Similar to Lemma 5, the probability to create one of the at least $\left(\begin{array}{l}4 \\ 3\end{array}\right) \mathrm{cn}$ subsets of size 3 of (the loosely connected) 4-cliques is lower bounded by $\left(\begin{array}{l}4 \\ 3\end{array}\right) c n \cdot p^{6}(1-p)^{n-6}$, while no maximum 4-clique is generated. And again similar to Lemma 5 , an expected number of at most $\mathcal{O}(1+n p)$ repetitions is sufficient to create the 4-clique prior to a maximal 3 -clique. This leads to an expected number of $\mathcal{O}\left(e^{n p} /\left(n p^{6}\right)\right)$. $\mathcal{O}(1+n p)=\mathcal{O}\left(e^{n p}(1+n p) /\left(n p^{6}\right)\right)$ steps, in total.

Part 2. If the element represents the 0 -clique, (a) with a probability of at least $2 c n \cdot p^{1}(1-p)^{n-1}$ a 1-cliques $\{v\}$, where $v \in V_{3}^{\prime}$ is created and moreover, (b) a non-empty different clique is created with a probability of at most $(16 n-$ $29) \cdot p^{1}(1-p)^{n-1}$ by Lemma 2 . Hence, the probability to perform (a) prior to (b) is bounded by $\Omega(1)$. Afterwards, the probability to create a 2-clique on $V_{3}$ prior to (b) is bounded by $\frac{p^{1}(1-p)^{n-1}}{p^{1}(1-p)^{n-1}+(16 n-29) \cdot p^{2}(1-p)^{n-2}}=\Omega(1 /(1+n p))$. Finally, the probability is also bounded by $\Omega(1 /(1+n p))$ to create a 3 -clique on $V_{3}$ prior to (b). Thus, a 3 -clique on $V_{3}$ is generated with probability $\Omega(1) \cdot \Omega(1 /(1+n p)) \cdot \Omega(1 /(1+$ $n p))=\Omega\left(1 /(1+n p)^{2}\right)$. Afterwards, the probability is upper bounded by $(12 n-24) \cdot p^{6}(1-p)^{n-6}$ to create a 3 -clique which is contained in a 4-clique or a 4-clique itself. This leads to an expected number of $\Omega\left(1 /(1+n p)^{2}\right) \cdot \Omega\left(e^{n p} /\left(n p^{6}\right)\right)=$ $\Omega\left(e^{n p} /\left(n p^{6}(1+n p)^{2}\right)\right)$ steps, in total.

\section{MULTI-START STRATEGY}

Let us define the simple $(1+1)$ RRLS $_{t}$, where the $(1+1)$ RLS is restarted after $t$ steps [7].

\section{Algorithm 7. $(\mathbf{1}+\mathbf{1}) \mathbf{R R L S}_{t}$}

1. Let $x:=0^{n}$ and $\ell:=0$.

2. Create $y$ by flipping one bit in $x$ uniformly at random.

3. If $f(y) \geq f(x)$ let $x:=y$.

4. Let $\ell:=\ell+1$. If $\ell=t$, Goto 1 . Otherwise, Goto 2 .

We observe the $(1+1) \operatorname{RRLS}_{\infty}$ behaves equivalently to the $(1+1)$ RLS and the $(1+1)$ RRLS $_{t}$ is not able to create a clique of size more than $t$. Hence, we disregard values $t \leq 3$.

Let us investigate planar graphs.

Lemma 8. Let $t \geq 4$ and $n$ be large enough. 
1. Let $G$ be a planar graph. The $(1+1) \mathrm{RRLS}_{t}$ creates a maximum clique of $G$ within an expected number of $\mathcal{O}\left(n^{4}+t \cdot n^{3}\right)$ steps.

2. There exist planar graphs $G$, where the $(1+1) \mathrm{RRLS}_{t}$ needs an expected number of $\Omega\left(n^{4}+t \cdot n^{3}\right)$ steps to create a maximum clique of $G$.

The optimal parameters for the $(1+1)$ RRLS $_{t}$ are $4 \leq t=$ $\mathcal{O}(n)$, where the expected number of steps is bounded by $\Theta\left(n^{4}\right)$, and the $(1+1) \mathrm{RRLS}_{t}$ is efficient, iff $4 \leq t=n^{\mathcal{O}(1)}$.

Proof. Part 1. Let $C$ be a maximum clique of $G$. With a probability of at least $\prod_{i=0}^{|C|-1}(|C|-i) / n=\Omega\left(1 / n^{3}\right)$ a subset of $C$ of size $|C|-1 \leq 3$ is generated within the first three steps. Afterwards, the probability that in the remaining $t-3$ steps a $|C|$-clique is created prior to a restart is lower bounded by $1-(1-1 / n)^{t-3}$ since one 1-bit flip creates $C$. We distinguish the cases $t \leq n$ (Case 1) and $t>n$ (Case 2). Case 1. We observe $(1-\delta)^{d} \leq 1-\delta \cdot d / 2$ for $0 \leq d \leq 1 / \delta$. Therefore, it holds $1-(1-1 / n)^{t-3}=\Omega(t / n)$. Hence, a phase between restarts is with probability $\Omega\left(1 / n^{3}\right) \cdot \Omega(t / n)=$ $\Omega\left(t / n^{4}\right)$ successful.

Case 2. We observe $1-(1-1 / n)^{t-3} \geq 1-e^{-(t-3) / n}=$ $\Omega(1)$. Hence, a phase between restarts is successful with probability $\Omega\left(1 / n^{3}\right) \cdot \Omega(1)=\Omega\left(1 / n^{3}\right)$.

Thus, an expected number of $\mathcal{O}\left(n^{4} / t+n^{3}\right)$ restarts is sufficient to generate a maximum clique. This leads to an expected number of at most $t \cdot \mathcal{O}\left(n^{4} / t+n^{3}\right)=\mathcal{O}\left(n^{4}+t \cdot n^{3}\right)$ steps, in total.

Part 2. We define the investigated planar graph $G$. Therefore, let $\ell:=\lfloor(n-4) / 6\rfloor$. Partition the vertices $V$ in $V_{0}:=$ $\left\{v_{1}, \ldots, v_{4}\right\}$ and $V_{i}:=\left\{v_{5+(i-1) \ell}, \ldots, v_{4+i \ell}\right\}, 1 \leq i \leq 6$. Let $e_{1}:=\left\{v_{1}, v_{2}\right\}, e_{2}:=\left\{v_{1}, v_{3}\right\}, e_{3}:=\left\{v_{1}, v_{4}\right\}, e_{4}:=$ $\left\{v_{2}, v_{3}\right\}, e_{5}:=\left\{v_{2}, v_{4}\right\}, e_{6}:=\left\{v_{3}, v_{4}\right\}$ and moreover, let $E_{i}:=\left\{\{u, w\},\{v, w\} \mid w \in V_{i}, e_{i}=\{u, v\}\right\}, 1 \leq i \leq 6$. The graph $G$ consists of the edges $\bigcup_{i=1}^{6} E_{i} \cup\left\{e_{i}\right\}$. The only maximum clique of $G$ is $V_{0}$. At the beginning of each phase between restarts with probability $4 / n$ a 1 -clique is generated which is a subset of $V_{0}$. Hence, with probability $3 /(3+3 \ell)=\mathcal{O}(1 / n)$ a 2 -clique and afterwards, with probability $2 /(2+\ell)=\mathcal{O}(1 / n)$ a 3 -clique which are subsets of $V_{0}$ are generated prior to a subset containing a vertex of $V \backslash V_{0}$. Thus, after a restart with probability $4 / n \cdot \mathcal{O}(1 / n) \cdot \mathcal{O}(1 / n)=$ $\mathcal{O}\left(1 / n^{3}\right)$ a 3 -clique on $V_{0}$ is created and otherwise an element such a restart is necessary. To the best after three steps a 3 -clique on $V_{0}$ is generated and within the remaining $t-3$ steps a particular 1-bit flip creates $V_{0}$. The probability for this 1-bit flip is upper bounded by $\min \{(t-3) / n, 1\}$. This leads to an expected number of $\Omega\left(n^{3} \cdot(n / t+1)\right)$ phases until it is successful. Since the length of a phase equals $t$ and all excepted the last phase is not successful, this leads to an expected number of at least $\left(\Omega\left(n^{4} / t+n^{3}\right)-1\right) \cdot t=\Omega\left(n^{4}+t \cdot n^{3}\right)$ steps, in total.

Let us investigate random planar graphs.

LEMMA 9. Let $t \geq 4$ and $n$ be large enough. The following holds with probability $1-e^{-\Omega(n)}$ and in expectation (w.r.t. the random input).

1. The $(1+1) \operatorname{RRLS}_{t}$ creates a maximum clique of $\mathcal{G}_{n}$ within an expected (w.r.t. the random bits of the algorithm) number of $\mathcal{O}\left(n^{3} / t^{2}+t\right)$ steps.

2. The $(1+1) \mathrm{RRLS}_{t}$ needs an expected (w.r.t. the random bits of the algorithm) number of $\Omega\left(n^{3} / t^{2}+t\right)$ steps to create a maximum clique of $\mathcal{G}_{n}$.
The optimal parameters for the $(1+1) \operatorname{RRLS}_{t}$ are $t=\Theta(n)$, where the expected number of steps is bounded by $\Theta(n)$, and the $(1+1) \operatorname{RRLS}_{t}$ is efficient, iff $4 \leq t=n^{\mathcal{O}(1)}$. We remark $t=4$ is an optimal parameter for planar graphs, but far away from being optimal for random planar ones.

Proof. By Lemma 3 with a failure probability of at most $e^{-c n}+e^{-c n}=e^{-\Omega(n)}$, for a constant $c>0$, the random planar graph $\mathcal{G}_{n}$ contains at least $c n$ loosely connected 3and 4-cliques. Let us assume that this holds in the following. Part 1. At the beginning of a phase between restarts with a probability of at least $3 \mathrm{cn} / n=\Omega(1)$ a 1-clique on $V_{4}^{\prime}$ is generated. Let $C$ be the loosely connected 4 -clique the 1clique is contained in. Let us partition the remaining $t-1$ steps in three subphases of length $(t-1) / 3 \geq 1$. Let a subset of size $\ell<4$ of $C$ be the current element. Similar to the proof of Part 1 of Lemma 8, the probability to create in one subphase a subset of $C$ of size $\ell+1$ is lower bounded by $\Omega(t / n)$, if $t \leq n$, and $\Omega(1)$, if $t>n$. Thus, with probability $\Omega\left(t^{3} / n^{3}\right)$, if $t \leq n$, and $\Omega(1)$, if $t>n$, in each of the three phases is successful and $C$ is generated. Thus, an expected number of $\mathcal{O}\left(n^{3} / t^{3}+1\right)$ restarts is sufficient to generate a maximum clique. In total, this leads to an expected number of $t \cdot \mathcal{O}\left(n^{3} / t^{3}+1\right)=\mathcal{O}\left(n^{3} / t^{2}+t\right)$ steps, in total.

Part 2. We distinguish $t \leq n$ (Case 1) and $t>n$ (Case 2).

Case 1. Let us lower bound the probability to create a maximum clique in one phase. Let $x_{(\ell)}, 0 \leq \ell \leq 4$, denote a $\ell$-clique. We observe a 4 -clique is generated by a sequence of cliques $x_{(0)}, x_{(1)}, x_{(2)}, x_{(3)}, x_{(4)}$. The succeeding clique is created with probability $1 / n$. The clique $x_{(1)}$ is created in the first step and the others in the next $t-1$ steps. Moreover, the number of such sequences is upper bounded by $4 \cdot 3 \cdot 2 \cdot 1$. $(6 n-12) \leq 144 n$ by Lemma 2 . Within $t$ steps the probability to generate a 4 -clique is upper bounded by

$$
144 n \cdot 1 / n \cdot\left(\begin{array}{c}
t-1 \\
3
\end{array}\right) \cdot 1 / n^{3} \leq 144 \cdot t^{3} / 3 ! \cdot 1 / n^{3}=t^{3} /\left(24 n^{3}\right) \quad .
$$

This leads to an expected number of $24 n^{3} / t^{3}$ phases until a 4 -clique is generated. Since the length of a phase equals $t$ and all excepted the last phase is not successful, this leads to an expected number of at least $\left(24 n^{3} / t^{3}-1\right) \cdot t \geq 23 n^{3} / t^{3} \cdot t=$ $\Omega\left(n^{3} / t^{2}\right)$ steps, where the inequality holds since $t \leq n$.

Case 2. We have seen $\left|V_{3}\right| \geq 3 \mathrm{cn}$. Thus, with a probability of at least $3 c$ a 1-clique on $V_{3}$ is generated initially. This leads to an expected number of $\Omega(t)$ steps.

The proposed result follows since $\Omega\left(n^{3} / t^{2}+t\right)=\Omega\left(n^{3} / t^{2}\right)$ for $t \leq n$ and $\Omega\left(n^{3} / t^{2}+t\right)=\Omega(t)$ for $t>n$.

\section{LARGE POPULATION}

Let us define the simple $(\mu+1)$ RLS which population consists of $\mu$ elements, where after the initialization no duplicates are inserted [12].

\section{Algorithm 10. $(\boldsymbol{\mu}+\mathbf{1}) \mathbf{R L S}$}

1. Let the population $\mathcal{P}$ consist of $\mu$ copies of $x:=0^{n}$.

2. Choose an individual $x$ of the population $\mathcal{P}$ uniformly at random. Create $y$ by flipping one bit in $x$ uniformly at random.

3. If $y \notin \mathcal{P}$, then let $z \in \mathcal{P} \cup\{y\}$ be randomly chosen among those individuals with the worst $f$-value and let the population be $\mathcal{P} \cup\{y\} \backslash\{z\}$.

4. Goto 2 
We observe the $(\mu+1)$ RLS, where $\mu=1$, behaves equivalently to the $(1+1)$ RLS on the maximum clique problem.

Let us extend the observations made for the $(1+1)$ RLS in the introduction to larger populations. Therefore, let us take a closer look to the graph with the only edge $\left\{v_{1}, v_{2}\right\}$ again. The $(\mu+1)$ RLS, where $\mu \leq n-2$, needs an infinite expected number of steps to find the maximum clique of this graph since with positive probability after $\mu$ steps the population consists of the cliques $\left\{v_{3}\right\}, \ldots,\left\{v_{2+\mu}\right\}$. Therefore, it is sufficient to consider at least linear population sizes.

Let us investigate planar graphs.

Lemma 11. Let $\mu \geq 16 n-29$ and $n$ be large enough.

1. Let $G$ be a planar graph. The $(\mu+1)$ RLS creates a maximum clique of $G$ within an expected number of $\mathcal{O}(\mu n)$ steps.

2. There exist planar graphs $G$, where the $(\mu+1)$ RLS needs an expected number of $\Omega(\mu n)$ steps to create a maximum clique of $G$.

The optimal parameters for the $(1+1) \mathrm{EA}_{p}$ are $16 n-29 \leq$ $\mu=\mathcal{O}(n)$, where the expected number of steps is bounded by $\Theta\left(n^{2}\right)$, and the $(1+1) \mathrm{EA}_{p}$ is efficient, iff $16 n-29 \leq \mu=$ $n^{\mathcal{O}(1)}$.

Proof. Part 1. We observe if a clique is created not contained in the population before, then this element is also inserted into the population and a copy of $0^{n}$ is removed. This holds since no duplicates are inserted into the population after the initialization and by Lemma 2 the population size is at least as large as the number of cliques in $G$. Let $C$ be a maximum clique of $G$. If the population contains a proper subset of $C$, then with a probability of at least $1 /(\mu n)$ a larger subset of $C$ is created and inserted into the population for sure. This leads to an expected number of at most $\sum_{\ell=0}^{|C|-1} \mu n=\mathcal{O}(\mu n)$ steps, in total.

Part 2. We define the investigated planar graph $G$. Therefore, let $E:=\left\{\left\{v_{1}, v_{2}\right\}\right\}$. While the maximum clique is not found, the probability is upper bounded by $2 /(\mu n)$ to create it since the population contains at most all other cliques and only a 1-bit flip, which has probability $1 / n$ to be performed, of the cliques $\left\{v_{1}\right\}$ or $\left\{v_{2}\right\}$, which have probability $2 / \mu$ to be selected, generates the maximum clique. This leads to an expected number of $\Omega(\mu n)$ steps, in total.

We remark that after an expected number of $\mathcal{O}\left(\mu n^{2}\right)$ steps the population contains a copy of each clique.

Let us investigate random planar graphs.

LEMMA 12. Let $\mu \geq 16 n-29$ and $n$ be large enough. The following holds with probability $1-e^{-\Omega(n)}$ and in expectation (w.r.t. the random input).

1. The $(\mu+1)$ RLS creates a maximum clique of $\mathcal{G}_{n}$ within an expected (w.r.t. the random bits of the algorithm) number of $\mathcal{O}\left(\mu n^{2 / 3}\right)$ steps.

2. The $(\mu+1)$ RLS needs an expected (w.r.t. the random bits of the algorithm) number of $\Omega\left(\mu n^{2 / 3}\right)$ steps to create a maximum clique of $\mathcal{G}_{n}$.

The optimal and the parameters, where the $(\mu+1)$ RLS is efficient, are the same than on planar graphs.

Proof. By Lemma 3 with a failure probability of at most $e^{-c n}+e^{-c n}=e^{-\Omega(n)}$, for a constant $c>0$, the random planar graph $\mathcal{G}_{n}$ contains at least $c n$ loosely connected 3and 4-cliques. Let us assume that this holds in the following. Part 1. By Lemma 2 the number of 0 -cliques in the population is always lower bounded by $\mu-(10 n-17)$ while no 4 -clique is generated. Hence, with a probability of at least $\frac{\mu-10 n+17}{\mu} \geq 1 / 4$ a 0 -clique is selected for mutation. If the population contains $0 \leq \ell<n 1$-cliques, then with a probability of at least $1 / 4 \cdot(n-\ell) / n$ a 1 -clique is created not contained in the population before. Hence, after an expected number of $4 \cdot \sum_{\ell=0}^{n-1} n /(n-\ell)=\mathcal{O}(n \log n)$ steps the population contains all 1-cliques. In this situation and while there exist less than $n^{2 / 3}$ 2-cliques on $V_{4}$, with a probability of at least $\left(4 c n-n^{2 / 3}\right) /(\mu n)=\Omega(1 / \mu)$ a 2-clique on $V_{4}$ is generated. Thus, at least $n^{2 / 3} 2$-cliques on $V_{4}$ are created within an expected number of $n^{2 / 3} \cdot \mathcal{O}(\mu)=\mathcal{O}\left(\mu n^{2 / 3}\right)$ steps. Similarly, within an expected number of $\mathcal{O}\left(\mu n^{2 / 3}\right)$ further steps at least $n^{1 / 3} 3$-cliques on $V_{4}$ are created. Finally, a maximum clique is generated within an expected number of at most $\mu / n^{1 / 3} \cdot n=\mathcal{O}\left(\mu n^{2 / 3}\right)$ steps. This leads to an expected number of $\mathcal{O}(n \log n)+\mathcal{O}\left(\mu n^{2 / 3}\right)+\mathcal{O}\left(\mu n^{2 / 3}\right)+\mathcal{O}\left(\mu n^{2 / 3}\right)=$ $\mathcal{O}\left(\mu n^{2 / 3}\right)$ steps, in total.

Part 2. Let us lower bound the probability to create a maximum clique in the first $\mu n^{2 / 3} / 6$ steps. Let $x_{(\ell)}$ and $y_{(\ell)}, 1 \leq \ell \leq 4$, denote $\ell$-cliques. We observe that a 4 clique is generated either directly by a sequence of cliques $\ldots, x_{(1)}, x_{(2)}, x_{(3)}, x_{(4)}$ (Case 1) or indirectly by a sequence of cliques ..., $y_{(2)}, y_{(3)}, x_{(2)}, x_{(3)}, x_{(4)}$ (Case 2). The succeeding clique is created with probability $1 /(\mu n)$.

Case 1. Let $x_{(1)}$ be an element of the population. The number of such sequences is upper bounded by $4 \cdot 3 \cdot 2 \cdot 1$. $(6 n-12) \leq 144 n$ by Lemma 2 . Hence, within $\mu n^{2 / 3} / 6$ steps the probability to generate a 4 -clique is upper bounded by

$$
144 n \cdot\left(\begin{array}{c}
\frac{\mu n^{2 / 3}}{6} \\
3
\end{array}\right) \cdot\left(\frac{1}{\mu n}\right)^{3} \leq \frac{\left(\mu^{3} n^{2}\right) \cdot(144 n)}{\left(3 ! \cdot 6^{3}\right) \cdot\left(\mu^{3} n^{3}\right)}=\frac{1}{9} .
$$

Case 2. Let $y_{(2)}$ be an element of the population and let $\ell_{y_{(3)}}$ denote the number of 4-cliques the 3-clique $y_{(3)}$ has at least one common edge with. The sum of the degrees of the three vertices of $y_{(3)}$ is lower bounded by $\ell_{y_{(3)}} / 7$. Otherwise, by Lemma 2 the subgraph on $y_{(3)}$ and its adjacent vertices consists of at most $6 \cdot\left(3+\ell_{y_{(3)}} / 7-1\right)-12=6 \ell_{y_{(3)}} / 7<\ell_{y_{(3)}}$ maximum cliques. Let us investigate all 3 -cliques which have at least one common edge with at least $\ell_{1}$ but less than $\ell_{2} \leq 6 n$ maximum cliques. Since the sum of all vertices' degrees in the graph is upper bounded by $2 \cdot(3 n-6) \leq 6 n$ and by Lemma 2 , the number of such 3 -cliques is upper bounded by $\min \left\{6 n /\left(\ell_{1} / 7\right), 6 n-12\right\} \leq \min \left\{42 n / \ell_{1}, 6 n\right\}$. Hence, the number of sequences with an $y_{(3)}$, where $\ell_{1} \leq \ell_{y_{(3)}}<\ell_{2}$, is upper bounded by $\min \left\{42 n / \ell_{1}, 6 n\right\} \cdot 3 \cdot 2 \cdot 2 \cdot 1 \cdot \ell_{2}=$ $\min \left\{42 n / \ell_{1}, 6 n\right\} \cdot 12 \cdot \ell_{2}$. We distinguish the cases $\ell_{1}=n^{2 / 3}$, $\ell_{2}=6 n$ (Case 2.a), $\ell_{1}=n^{1 / 3}, \ell_{2}=n^{2 / 3}$ (Case 2.b), and $\ell_{1}=1, \ell_{2}=n^{1 / 3}$ (Case 2.c) and we consider $\mu n^{2 / 3} / 6$ steps. The probability to generate a 4 -clique is upper bounded by: Case 2.a

$\frac{42 n}{n^{2 / 3}} \cdot 12 \cdot 6 n \cdot\left(\begin{array}{c}\frac{\mu n^{2 / 3}}{6} \\ 4\end{array}\right) \cdot\left(\frac{1}{\mu n}\right)^{4} \leq \frac{\left(\mu^{4} n^{8 / 3}\right) \cdot\left(3024 n^{4 / 3}\right)}{\left(4 ! \cdot 6^{4}\right) \cdot\left(\mu^{4} n^{4}\right)}=\frac{7}{72}$

Case 2.b

$\frac{42 n}{n^{1 / 3}} \cdot 12 \cdot n^{2 / 3} \cdot\left(\begin{array}{c}\mu n^{2 / 3} \\ 6\end{array}\right) \cdot\left(\frac{1}{\mu n}\right)^{4} \leq \frac{\left(\mu^{4} n^{8 / 3}\right) \cdot\left(504 n^{4 / 3}\right)}{\left(4 ! \cdot 6^{4}\right) \cdot\left(\mu^{4} n^{4}\right)}=\frac{7}{432}$ 
Case 2.c

$6 n \cdot 12 \cdot n^{1 / 3} \cdot\left(\begin{array}{c}\frac{\mu n^{2 / 3}}{6} \\ 4\end{array}\right) \cdot\left(\frac{1}{\mu n}\right)^{4} \leq \frac{\left(\mu^{4} n^{8 / 3}\right) \cdot\left(72 n^{4 / 3}\right)}{\left(4 ! \cdot 6^{4}\right) \cdot\left(\mu^{4} n^{4}\right)}=\frac{1}{432}$

Thus, with a probability of at least $1-\left(\frac{1}{9}+\frac{7}{72}+\frac{7}{432}+\frac{1}{432}\right) \geq$ $\frac{3}{4}$ no maximum clique is generated within $\mu n^{2 / 3} / 6$ steps. This leads to an expected number of at least $3 / 4 \cdot \mu n^{2 / 3} / 6=$ $\Omega\left(\mu n^{2 / 3}\right)$ steps, in total.

\section{NON-ELITISM SELECTION}

Let us define the simple $\mathrm{MA}_{T}$ occasionally accepting elements with larger function values than the current one [8].

\section{Algorithm 13. MA}

1. Let $x:=0^{n}$.

2. Create $y$ by flipping one bit in $x$ uniformly at random.

3. With probability $\min \left\{e^{T \cdot(f(y)-f(x))}, 1\right\}$, let $x:=y$.

4. Goto 2 .

We observe the $\mathrm{MA}_{\infty}$ behaves equivalently to the $(1+1)$ RLS. Moreover, the Metropolis algorithm $\mathrm{MA}_{T}$ does not accept elements which do not represent cliques. Hence, a by one large clique is accepted with probability 1 and a by one smaller clique is accepted with probability $e^{-T}=: 1 / \lambda$.

Let us investigate planar graphs.

LEMma 14. Let $T \geq 0$ and $n$ be large enough.

1. Let $G$ be a planar graph. The $\mathrm{MA}_{T}$ creates a maximum clique of $G$ within an expected number of $\mathcal{O}\left(n^{2} e^{4 T}\right)$ steps.

2. There exist planar graphs $G$, where the $\mathrm{MA}_{T}$ needs an expected number of $\Omega\left(n^{2} e^{T}\right)$ steps to create a maximum clique of $G$.

The optimal parameters for the $\mathrm{MA}_{T}$ are $0 \leq T=\mathcal{O}(1)$, where the expected number of steps is bounded by $\Theta\left(n^{2}\right)$, and the $\mathrm{MA}_{T}$ is efficient, iff $0 \leq T=\mathcal{O}(\log n)$.

Proof. Part 1. We consider the Markov chain induced by the $\mathrm{MA}_{T}$ on $G$. The state space is the set of all cliques in $G$. If state $C^{+}$and $C^{-}$are a by one larger superset and smaller subset respectively of a state $C$, then the probability of a transition from $C$ to $C^{+}$is $1 / n$ and $1 /(\lambda n)$ to $C^{-}$. All other non-loop transitions have probability 0 . Thus, the expected number of steps until a transition occurs is upper bounded by $\lambda n$. Let us consider the transition steps only. Equivalently, we can investigate a random walk on the following connected and undirected, but weighted graph $W$. The vertices are the states and the edges $E_{W}$ are the non-zero transitions of the Markov chain considered. The weight $r_{\{v, w\}}$ for an edge $\{v, w\}$ between a $\ell$ - and $(\ell+1)$ clique is $\lambda^{\ell}$. We recall the probability to transition from $v$ to $w$ equals $r_{\{v, w\}} / \sum_{\{v, u\} \in E_{W}} r_{\{v, u\}}$. Hence, $W$ consists of at most $\left|E_{W}\right| \leq 1 \cdot n+2 \cdot(3 n-6)+3 \cdot(6 n-12)+4 \cdot(6 n-12)=\mathcal{O}(n)$ edges. The maximal sum of the weights on a shortest path between any two vertices of $W$ is upper bounded by $\lambda^{3}+\lambda^{2}+$ $\lambda+1+1+\lambda+\lambda^{2}+\lambda^{3}=\mathcal{O}\left(\lambda^{3}\right)$. Since the weights are lower bounded by 1 , it holds $\sum_{\{v, w\} \in E_{W}} 2 / r_{\{v, w\}} \leq 2 \cdot\left|E_{W}\right|=$ $\mathcal{O}(n)$. By [2, Theorem 2] for any two vertices $v$ and $w$ the expected number of edges traversed by a random walk on $W$ starting in $v$, reaching $w$, and ending upton first reaching $v$ again, is upper bounded by $\mathcal{O}(n) \cdot \mathcal{O}\left(\lambda^{3}\right)=\mathcal{O}\left(n \lambda^{3}\right)$. Let us add the not state changing steps. This leads to an expected number of at most $\lambda n \cdot \mathcal{O}\left(n \lambda^{3}\right)=\mathcal{O}\left(n^{2} \lambda^{4}\right)$ steps, in total.

Part 2. We define the investigated planar graph $G$. Therefore, let $E:=\left\{\left\{v_{1}, v_{2}\right\}\right\}$. At least once a clique $\left\{v_{1}\right\}$ or $\left\{v_{2}\right\}$ has to be created. If the individual represents the 0-clique, with probability $2 / n$ such a 1 -clique is created. Otherwise, the 1-clique $\left\{v_{3}\right\}, \ldots,\left\{v_{n}\right\}$ is replaced by the 0 -clique within an expected number of $\lambda n$ steps. This leads to an expected number of at least $\lambda n \cdot(n / 2-1)=\Omega\left(n^{2} \lambda\right)$ steps, in total.

Let us investigate random planar graphs.

LEMmA 15. Let $T \geq 0$ and $n$ be large enough. The following holds with probability $1-e^{-\Omega(n)}$ and in expectation (w.r.t. the random input).

1. The $\mathrm{MA}_{T}$ creates a maximum clique of $\mathcal{G}_{n}$ within an expected (w.r.t. the random bits of the algorithm) number of $\mathcal{O}\left(n e^{4 T}\right)$ steps.

2. The $\mathrm{MA}_{T}$ needs an expected (w.r.t. the random bits of the algorithm) number of $\Omega\left(n e^{T}\right)$ steps to create a maximum clique of $\mathcal{G}_{n}$.

The optimal and the parameters, where the $\mathrm{MA}_{T}$ is efficient, are the same than on planar graphs.

Proof. By Lemma 3 with a failure probability of at most $e^{-c n}+e^{-c n}=e^{-\Omega(n)}$, for a constant $c>0$, the random planar graph $\mathcal{G}_{n}$ contains at least $c n$ loosely connected 3and 4-cliques. Let us assume that this holds in the following.

Part 1. Similar to the proof of Part 1 of Lemma 14 we consider the Markov chain induced by the $\mathrm{MA}_{T}$ on $G$. It is irreducible and aperiodic for $n \geq 5$. Thus, the process is ergodic and possesses a unique stationary distribution $\pi$, where for the state $S$ which represents the 0-clique it holds $\pi(S)=1 / \sum_{C} \lambda^{|C|}$ by [8, page 350]. By [11, Theorem 6.2] the expected number of steps starting in $S$ and ending upon the first return to $S$, equals $1 / \pi(S)=\sum_{C} \lambda^{|C|} \leq(16 n-29)$. $\lambda^{4}=\mathcal{O}\left(n \lambda^{4}\right)$ by Lemma 2 . Moreover, similar to Lemma 9 , starting in $S$ with a probability of at least $3 c \cdot 3 \lambda /(3 \lambda+1)$. $2 \lambda /(2 \lambda+2) \cdot \lambda /(\lambda+3)=\Omega(1)$ a 4 -clique is generated prior to return to $S$. Thus, an expected number of $\mathcal{O}(1)$ returns to $S$ is sufficient until a 4 -clique is created. This leads to an expected number of $\mathcal{O}(1) \cdot \mathcal{O}\left(n \lambda^{4}\right)=\mathcal{O}\left(n \lambda^{4}\right)$ steps, in total. Part 2. Similar to the proof of Part 2 of Lemma 9, with a probability of at least $2 c$ a 1 -clique on $V_{3}^{\prime}$ is created in the first step. Afterwards, at least once a worsening has to be accepted, which needs an expected number of at least $\lambda n / 3$ steps. This leads to an expected number of at least $2 c \cdot n \lambda / 3=\Omega(n \lambda)$ steps, in total.

\section{BLACK-BOX COMPLEXITY}

We have seen that the $\mathrm{MA}_{1}$ - asymptotically a most efficient heuristic investigated on the planar graph models needs $\Theta\left(n^{2}\right)$ expected steps to find a maximum clique in planar graphs and in random planar graphs it needs $\Theta(n)$ expected steps. The question arises if any optimizer can be asymptotically more efficient, namely let us lower bound the expected number of steps to find a maximum clique in planar and random planar graphs by any general search heuristic.

One characteristic of all general search heuristics is that they gather information about the problem instance - the problem itself is (probably) known in advance - by querying 
one search point after the other to a so-called black-box. At time $t$ the next query $x_{t}$ is determined by a black-box algorithm with knowledge about the whole history of the pairs of previously queried elements and their function values.

\section{Algorithm 16. Black-Box Algorithm}

Step $t \geq 1$ : Depending on $\left(x_{1}, f\left(x_{1}\right)\right), \ldots,\left(x_{t-1}, f\left(x_{t-1}\right)\right)$ determine a probability distribution on $\{0,1\}^{n}$, choose $x_{t}$ according to this distribution, query $x_{t}$, and receive its function value $f\left(x_{t}\right)$ from the black-box.

A black-box algorithm which determines deterministically or randomly the next query is called a deterministic or randomized black-box algorithm respectively. We remark the class of randomized black-box algorithms includes the class of deterministic ones. Considering all (randomized) black-box algorithms allows us to obtain general lower bound on the complexity of a problem for search heuristics. This framework was introduced by Droste, Jansen, and Wegener [4]. In contrast to investigations of a specific optimizer, it is not meaningful to consider the black-box complexity for a single function $f$. In such a scenario always an efficient black-box algorithm exists. It just queries an optimal element of $f$ within the first step. Therefore, classes $\mathcal{F}$ of functions are considered, whereof the particular function $f \in \mathcal{F}$ is randomly chosen. Let us investigate the following (deterministic) black-box algorithms BBA and BBA*

\section{Algorithm 17. BBA and BBA*}

1. Let $A_{1}:=\emptyset, \ldots, A_{n}:=\emptyset$, and $i:=1$.

2. For $j:=i+1, \ldots, n$ query $\left\{v_{i}, v_{j}\right\}$. If the function value equals 2 , then $A_{i}:=A_{i} \cup\left\{v_{j}\right\}, A_{j}:=A_{j} \cup\left\{v_{i}\right\}$.

$\star$. If $\left|A_{i}\right|=3$, then query $A_{i} \cup\left\{v_{i}\right\}$.

$\mathrm{BBA}^{\star}$ only

3. Let $i:=i+1$. If $i=n$, then Goto 2, else Goto 4 .

4. Calculate a maximum clique $C$ (without performing any query; for planar graphs efficiently similar to [5] and for general graphs similar to [13]) and query $C$.

At step 4 no query except the one of $C$ is needed since $A_{i}$, $1 \leq i \leq n$, consists of all vertices adjacent to $v_{i}$. Namely, if the function value of a query $\left\{v_{i}, v_{j}\right\}$ equals 2 , then the edge $\left\{v_{i}, v_{j}\right\}$ exists and otherwise, the function value equals $-\infty$ and the edge $\left\{v_{i}, v_{j}\right\}$ does not exist. Hence, $G$ is uncovered.

Let us investigate (planar) graphs. Therefore, let $\mathcal{E}_{n}$ consist of all $\left(\begin{array}{l}n \\ 2\end{array}\right)$ (planar) graphs with a single edge on $n$ vertices.

LEMMA 18.

1. Let $G$ be a (planar) graph. The BBA creates a maximum clique of $G$ within at most $\left(\begin{array}{c}n \\ 2\end{array}\right)+1=\mathcal{O}\left(n^{2}\right)$ steps.

2. Each black-box algorithm needs an expected number of at least $\left(\left(\begin{array}{c}n \\ 2\end{array}\right)+1\right) / 2=\Omega\left(n^{2}\right)$ steps to create a maximum clique of $G \in \mathcal{E}_{n}$.

Proof. Part 1. The proposed result follows directly since the BBA performs totally at most $\left(\begin{array}{l}n \\ 2\end{array}\right)$ queries in step 2 and additionally one query in step 4 .

Part 2. We observe a query of an element $x$ leads to function values $-\infty$, if $|x| \geq 3$, and $|x|$, if $|x| \leq 1$. Moreover, for each $x$, where $|x|=2$, a single function of $\mathcal{E}_{n}$ leads to function value 2 , for all others it is $-\infty$. Thus, each black-box algorithm has to search for the "needle in a haystack" of size $\left(\begin{array}{c}n \\ 2\end{array}\right)$. By $[4$, Theorem 1] the expected number of queries of each black-box algorithm is lower bounded by $\left(\left(\begin{array}{l}n \\ 2\end{array}\right)+1\right) / 2$.
Let us investigate random planar graphs.

Lemma 19. The following holds with probability $1-e^{-\Omega(n)}$ and in expectation (w.r.t. the random input).

1. The $\mathrm{BBA}^{\star}$ creates a maximum clique of $\mathcal{G}_{n}$ within $\mathcal{O}(n)$ steps.

2. Each black-box algorithm needs an expected (w.r.t. the random bits of the algorithm) number of $\Omega(n)$ steps to generate a maximum clique of $\mathcal{G}_{n}$.

Proof. By Lemma 3 a fraction of at most $e^{-c n}$ planar graphs contains less than $\mathrm{cn}$ loosely connected 4-cliques.

Part 1. Let us investigate all planar graphs containing at least $c n$ loosely connected 4-cliques. We observe for each permutation $\pi$ on $1, \ldots, n$ and $\ell \geq 0, V_{4}^{\prime}$ equals $\left\{v_{i_{1}}, \ldots, v_{i_{\ell}}\right\}$ and $\left\{v_{\pi\left(i_{1}\right)}, \ldots, v_{\pi\left(i_{\ell}\right)}\right\}$ for the same fraction of planar graphs. Thus, having cycled the loop of step 2, , and 3 exactly $i<c n, c<1$, times for a fraction of at most

$$
\left(\begin{array}{c}
n-i \\
3 c n
\end{array}\right) /\left(\begin{array}{c}
n \\
3 c n
\end{array}\right)=\frac{(n-3 c n) \cdots(n-3 c n-i+1)}{n \cdots(n-i+1)} \leq\left(\frac{n-3 c n}{n-c n}\right)^{i} \leq(1-c)^{i}
$$

graphs no maximum clique is created in total in step $\star$. After the loop has been cycled $c n-1$ times for a fraction of at most $(1-c)^{c n-1} \leq e^{-c^{2} n-1}$ planar graphs no maximum clique is created. In this situation or if the planar graph contains less than $c n$ loosely connected 4-cliques, at most $\left(\begin{array}{l}n \\ 2\end{array}\right)+(n-1)+1 \leq n^{2}$ queries are performed until a maximum clique is created. This leads to an expected number of at $\operatorname{most} \sum_{i=0}^{c n-1}(1-c)^{i} \cdot(n-i)+\left(e^{-c^{2} n-1}+e^{-c n}\right) \cdot n^{2} \leq n / c+1=$ $\mathcal{O}(n)$ steps, in total.

Part 2. Let $x_{1}, \ldots, x_{n / 13}$ be an arbitrary sequence of elements queried within the first $n / 13$ steps. We prove that for a fraction of at least $1 / 2$ of all planar graphs investigated the function values $\left|x_{i}\right|$ are obtained for all elements $x_{i}$, where $\left|x_{i}\right| \leq 1$ and $-\infty$ is obtained, where $\left|x_{i}\right| \geq 2$. This is obviously true for $\left|x_{i}\right| \leq 1$ and $\left|x_{i}\right| \geq 5$ and all planar graphs. Let us investigate the elements $x_{i}$, where $2 \leq\left|x_{i}\right| \leq 4$. Again, we observe for each permutation $\pi$ on $1, \ldots, n$ and $\ell \geq 0,\left\{v_{i_{1}}, \ldots, v_{i_{\ell}}\right\}$ and $\left\{v_{\pi\left(i_{1}\right)}, \ldots, v_{\pi\left(i_{\ell}\right)}\right\}$ are $\ell$-cliques for the same fraction of planar graphs. By Lemma 2 the fraction of planar graphs,

- where $\left\{v_{1}, v_{2}\right\}$ represents a clique is upper bounded by $(3 n-6) /\left(\begin{array}{c}n \\ 2\end{array}\right) \leq 6 / n$,

- where $\left\{v_{1}, v_{2}, v_{3}\right\}$ represents a clique is upper bounded by $(6 n-12) /\left(\begin{array}{l}n \\ 3\end{array}\right) \leq 37 / n^{2} \leq 6 / n$, and

- where $\left\{v_{1}, v_{2}, v_{3}, v_{4}\right\}$ represents a clique is upper bounded by $(6 n-12) /\left(\begin{array}{l}n \\ 4\end{array}\right) \leq 145 / n^{3} \leq 6 / n$.

The fraction of planar graphs, where either the maximum clique is smaller than two or a clique of size at least two is created within the first $n / 13$ steps, is upper bounded by $e^{-c n}+6 / n \cdot n / 13 \leq 1 / 2$. We have considered all possible sequences of elements queried. This leads to an expected number of at least $(n / 13+1) \cdot 1 / 2=\Omega(n)$ steps, in total.

\section{SEMI-RANDOM PLANAR GRAPHS}

As mentioned in the introduction, we investigate the semirandom planar graphs $\mathcal{G}_{n}^{+}, \mathcal{G}_{n}^{-}$, and $\mathcal{G}_{n}^{\star}$, where we choose $\varepsilon=c / 4$, for the constant $c>0$ mentioned in Lemma 3 . Here, we restrict to an optimal choice of the algorithms' parameter on planar and random planar graphs. 
THEOREM 20. The bounds on the expected (w.r.t. the random bits of the algorithm) number of steps to create a maximum clique of a semi-random planar graph - given in the following table - hold with probability $1-n^{-d}$, for any constant $d>0$, and in expectation (w.r.t. the random part of the input).

\begin{tabular}{|l||c|c|c|}
\hline & $\mathcal{G}_{n}^{+}$ & $\mathcal{G}_{n}^{-}$ & $\mathcal{G}_{n}^{\star}$ \\
\hline \hline$(1+1) \mathrm{EA}_{1 / n}$ & $\Theta\left(n^{5}\right)$ & $\Theta\left(n^{6}\right)$ & $\Theta\left(n^{5}\right)$ \\
\hline$(1+1) \mathrm{RRLS}_{n}$ & $\Theta(n)$ & $\mathcal{O}\left(n^{2}(\log n)^{2}\right)$ & $\Theta(n)$ \\
& & $\Omega\left(\frac{n^{2}(\log n)^{2}}{(\log \log n)^{2}}\right)$ & \\
\hline$(16 n+1) \mathrm{RLS}$ & $\Theta\left(n^{5 / 3}\right)$ & $\Theta\left(n^{2}\right)$ & $\Theta\left(n^{5 / 3}\right)$ \\
\hline MA $_{1}$ & $\Theta(n)$ & $\Theta\left(n^{2}\right)$ & $\Theta(n)$ \\
\hline black-box complex. & $\Omega(n)$ & $\Omega\left(n^{2}\right)$ & $\Omega(n)$ \\
\hline
\end{tabular}

Proof. By Lemma 3 with a failure probability of at most $e^{-c n}+e^{-c n} \leq n^{-d} / 3$ the random planar graph, which is modified by an adversary afterwards, contains at least $c n$ loosely connected 3 - and 4-cliques. Let $H_{\ell}$ be the graph described in the proof of Part 2 of Lemma 8 on $6 \ell+4$ vertices. Similar to [10, Proposition 4.4] the random planar graph on $n$ vertices contains at least one subgraph $H_{d_{1} \log n / \log \log n}$ with a failure probability of at most $n^{-d} / 3$, for a constant $d_{1}>0$ depending on $d$. Moreover, similar to $[10$, Proposition 4.5] the random planar graph on $n$ vertices consists of vertices with a degree of at most $d_{2} \log n$ only with a failure probability of at most $n^{-d} / 3$, for a constant $d_{2}>0$ depending on $d$. Let us assume that all this holds in the following.

Let us investigate a graph $\mathcal{G}_{n}^{+}$. The lower bounds follow by Part 2 of Lemmas 6, 9, 12, 15, and 19. Since no 4clique is removed, it is seen easily that the upper bounds for the $(1+1) \mathrm{EA}_{1 / n}$ and the $(16 n+1)$ RLS follow by Part 1 of Lemmas 6 and 12. Hence, since at most $3 n-6$ edges are inserted in the random planar graph by an adversary, the degree of at least $c n$ vertices of $V_{4}$ is at most $3 / c$, afterwards. Thus, starting with the 0 -clique the probability to generate a 4 -clique is lower bounded by $\Omega(1)$ for the $(1+1)$ RRLS $_{n}$ and the $\mathrm{MA}_{1}$. Similar to the proof of Part 1 of Lemmas 9 and 15 , this leads to the proposed results.

Let us investigate a graph $\mathcal{G}_{n}^{-}$. For the $(1+1) \mathrm{EA}_{1 / n}$, the $(16 n+1)$ RLS, the $\mathrm{MA}_{1}$, and the black-box complexity the lower and upper bounds follow by Lemmas 5, 11, 14, and 18 since the graphs described in the proofs of Part 2 are all producible by edge removal, if the random planar graph consists of at least one loosely connected 3- and 4-clique. Let us consider the $(1+1) \operatorname{RRLS}_{n}$. For the lower bound all edges except these of one subgraph $H_{d_{1}} \log n / \log \log n$ are removed. Similar to the proof of Part 2 of Lemma 8 a 3 -clique which is a subset of the 4-clique is created with probability $\mathcal{O}(1 / n)$. $\mathcal{O}(\log \log n / \log n) \cdot \mathcal{O}(\log \log n / \log n)=\mathcal{O}\left(\frac{(\log \log n)^{2}}{n(\log n)^{2}}\right)$. This leads to the proposed lower bound since a restart occurs after $n$ steps. For the upper bound we observe the maximal degree is upper bounded by $d_{2} \log n$. Similar to the proof of Part 1 of Lemma 8 with probability $\Omega(1 / n) \cdot \Omega(1 / \log n)$. $\Omega(1 / \log n) \cdot \Omega(1)=\Omega\left(\frac{1}{n(\log n)^{2}}\right)$ a maximum clique is created.

Let us investigate a graph $\mathcal{G}_{n}^{\star}$. The lower and upper bounds follow by Lemmas $6,9,12,15$, and 19 since only at most $2 \cdot c / 4 \cdot n=c n / 2$ of $c n$ loosely connected 3 - and 4 -cliques are modified by edge insertion or removal.

We remark the $\mathrm{BBA}^{\star}$ can be modified easily, such that it works asymptotically optimal for all semi-random planar graph models investigated here, too.

\section{CONCLUSIONS}

The optimization behavior of simple general randomized search heuristics for the maximum clique problem on planar and random planar graphs is considered rigorously in detail. These investigations are combined and extended for the algorithms' analysis on semi-random planar graphs. In this situation, the different strengths and weaknesses of four popular strategies to overcome local optima, namely local- vs. global-search, single- vs. multi-start, small vs. large population, and elitism vs. non-elitism are pointed out. Moreover, we have observed that in particular the Metropolis algorithm (with a carefully chosen fixed temperature) is an (asymptotically) optimal general heuristic for finding a maximum clique in planar and random planar graphs. Therefore, the black-box complexity for finding a maximum clique in planar and random planar graphs is determined, too. Future research will investigate further simple randomized search heuristics on further combinatorial optimization problems.

Acknowledgements. This research was supported by grant We 1066/11 from the German Research Foundation (DFG).

\section{REFERENCES}

[1] I. M. Bomze, M. Budinich, P. M. Pardalos, and M. Pelillo. Handbook of Combinatorial Optimization (supp. Vol. A), chapter The Maximum Clique Problem, pages 1-74. Kluwer, Dordrecht, 1999.

[2] A. K. Chandra, P. Raghavan, W. L. Ruzzo, R. Smolensky, and P. Tiwari. The electrical resistance of a graph captures its commute and cover times. In Proceedings 21th STOC, 1989.

[3] S. Droste, T. Jansen, and I. Wegener. On the analysis of the $(1+1)$ evolutionary algorithm. Theoretical Computer Science, 276:51-81, 2002.

[4] S. Droste, T. Jansen, and I. Wegener. Upper and lower bounds for randomized search heuristics in black-box optimization. Theory of Computing Systems, 2005. to appear.

[5] D. Eppstein. Subgraph isomorphism in planar graphs and related problems. Journal of Graph Algorithms and Applications, 3:1-27, 1999.

[6] U. Feige and J. Kilian. Heuristics for semi-random graph problems. Journal of Computer and System Science, 63:639-671, 2001.

[7] T. Jansen. On the analysis of dynamic restart strategies for evolutionary algorithms. In Proceedings PPSN VII, pages 33-43, 2002.

[8] M. Jerrum. Large cliques elude the Metropolis process. Random Structrures and Algorithms, 3:347-360, 1992.

[9] R. M. Karp. Reducibility among combinatorial problems. In Complexity of Computer Computations, pages 85-103, 1972.

[10] C. McDiarmid, A. Steger, and D. J. A. Welsh. Random planar graphs. Journal of Combinatorial Theory, Series B, 93:187-205, 2005.

[11] R. Motwani and P. Raghavan. Randomized Algorithms. Cambridge University Press, Cambridge, 1995.

[12] T. Storch. On the choice of the population size. In Proceedings GECCO 2004, pages 748-760, 2004.

[13] R. E. Tarjan and A. E. Trojanowski. Finding a maximum independent set. SIAM Journal on Computing, 6:537-546, 1977. 